\title{
Solution of the Least Squares Method problem of pairwise comparison matrices
}

\author{
Sándor Bozóki ${ }^{1}$
}

\begin{abstract}
The aim of the paper is to present a new global optimization method for determining all the optima of the Least Squares Method $(L S M)$ problem of pairwise comparison matrices. Such matrices are used, e.g., in the Analytic Hierarchy Process (AHP). Unlike some other distance minimizing methods, $L S M$ is usually hard to solve because of the corresponding nonlinear and non-convex objective function. It is found that the optimization problem can be reduced to solve a system of polynomial equations. Homotopy method is applied which is an efficient technique for solving nonlinear systems. The paper ends by two numerical example having multiple global and local minima.
\end{abstract}

\section{Introduction}

In Multi-Attribute Decision Making, one of the central questions is determining the weights of attributes (criteria) or the cardinal preferences of the alternatives (actions). Usually, the decision makers may be requested to tell neither the explicit weights of the criteria representing the importance, nor the cardinal preferences of the alternatives, but they can make pairwise comparisons. Condorcet [11] and Borda [3] used pairwise comparisons in the 18th century, as well as Weber and Fechner [17] in experimental psychology in the second half of the 19th century, then Thorndike [30] and Thurstone [31] in the 1920's.

The method of pairwise comparisons using ratio scale was developed by Thomas L. Saaty [28]. Using the Analytic Hierarchy Process (AHP), difficult decision problems can be broken into smaller parts by the hierarchical criterion-tree, one level of the tree can be handled by pairwise comparison matrices.

\footnotetext{
${ }^{1}$ Laboratory of Operations Research and Decision Systems Computer and Automation Institute, Hungarian Academy of Sciences, 1518 Budapest P.O. Box 63, Hungary, E-mail: bozoki@oplab.sztaki.hu

This research was supported in part by the Hungarian Scientific Research Fund, Grant No. OTKA K 60480.
} 
A pairwise comparison matrix $\mathbf{A}=\left[a_{i j}\right]_{i, j=1 . . n}$ is defined as

$$
\mathbf{A}=\left(\begin{array}{ccccc}
1 & a_{12} & a_{13} & \ldots & a_{1 n} \\
a_{21} & 1 & a_{23} & \ldots & a_{2 n} \\
a_{31} & a_{32} & 1 & \ldots & a_{3 n} \\
\vdots & \vdots & \vdots & \ddots & \vdots \\
a_{n 1} & a_{n 2} & a_{n 3} & \ldots & 1
\end{array}\right) \in \mathbb{R}_{+}^{n \times n}
$$

where $\mathbb{R}_{+}^{n \times n}$ denotes the class of positive $n \times n$ matrices, and for any $i, j=$ $1, \ldots, n$,

$$
a_{i j}>0, \quad a_{i j}=\frac{1}{a_{j i}} .
$$

The matrix element $a_{i j}$ expresses the relative importance or preference of $i$-th object compared to $j$-th object given by the decision maker $(i, j=$ $1,2, \ldots, n)$. For example, the first object is $a_{12}$ times more important/preferred than the second one.

An $\mathbf{A}=\left[a_{i j}\right]_{i, j=1 . . n}$ pairwise comparison matrix is called consistent, if it satisfies the following properties for all indices $i, j, k=1, \ldots, n$ :

$$
a_{i j}=\frac{1}{a_{j i}}, \quad a_{i j} a_{j k}=a_{i k} .
$$

In practical decision problems, pairwise comparison matrices given by the decision maker are not consistent. Based on the elements of the matrix, we want to find a weight vector $\mathbf{w}=\left(w_{1}, w_{2}, \ldots, w_{n}\right)^{T} \in \mathbb{R}_{+}^{n}$ representing the priorities of the objects where $\mathbb{R}_{+}^{n}$ is the positive orthant. The Eigenvector Method [28] and some distance minimizing methods such as the Least Squares Method [9, 24], Logarithmic Least Squares Method [14, 13, 12, 1], Weighted Least Squares Method [9, 2], Chi Squares Method [24], Logarithmic Least Absolute Values Method [10, 23] and Singular Value Decomposition [21] are some of the tools for computing the priorities of the alternatives.

After some comparative analyses [8, 29, 12, 32], one of the most comprehensive review was done by Golany and Kress [22]. They compared some scaling methods from the first 10-15 year history of pairwise comparison matrices by seven criteria and concluded that every method has advantages and weaknesses, none of them is prime.

Since $L S M$ problem was not solved fully, comparisons to other methods are restricted to a few specific examples. 
The aim of the paper is to present a new global optimization method for solving the $L S M$ problem for matrices up to the size $8 \times 8$ in order to ground for further research of comparisons to other methods and examining its real life application possibilities. It is shown that the first order necessary conditions of optimality can be transformed to a system of multivariate polynomials. Homotopy method is applied for finding all the solutions of the polynomial system, from which all the local and global optima of the objective function can be computed [6].

In the paper we study the Least Squares Method ( $L S M)$ which is a minimization problem of the Frobenius norm of $\left(\mathbf{A}-\mathbf{w} \frac{1}{\mathbf{w}}^{T}\right)$, where $\frac{1}{\mathbf{w}}^{T}$ denotes the row vector $\left(\frac{1}{w_{1}}, \frac{1}{w_{2}}, \ldots, \frac{1}{w_{n}}\right)$.

\section{Least Squares Method ( $L S M)$}

$$
\begin{aligned}
\min & \sum_{i=1}^{n} \sum_{j=1}^{n}\left(a_{i j}-\frac{w_{i}}{w_{j}}\right)^{2} \\
\text { s.t. } \quad & \sum_{i=1}^{n} w_{i}=1, \\
w_{i} & >0, \quad i=1,2, \ldots, n .
\end{aligned}
$$

$L S M$ is rather difficult to solve because the objective function is nonlinear and usually nonconvex, moreover, no unique solution exists [24, 25] and the solutions are not easily computable. Newton's method of successive approximation can be applied [16], but a good initial point is required.

Sufficient conditions of convexity of the $L S M$ objective function over the feasible set are given and branch-and-bound method is applied for the general case in [18].

The transformation of the first-order conditions of optimality into a multivariate polynomial system is described in Section 2. Properties of the polynomial sytems from the $L S M$ problem are characterized and three methods for finding all the positive real solutions of them are given in Section 3. Numerical examples of Section 4 are to illustrate the complexity of the $L S M$ problem as the dimension number increases. It is noted in Section 5 that the methods in Section 2 and 3 may be generalized into the case of incomplete pairwise comparison matrices. Two examples of Section 6 show that the number of solutions of the $L S M$ problem may be equal to or even twice as many as the dimension of the matrix. The paper ends by the conclusions of Section 7. 


\section{The $L S M$ problem}

Let $\mathbf{A}$ be an $n \times n$ matrix obtained from pairwise comparisons in the form

$$
\mathbf{A}=\left(\begin{array}{ccccc}
1 & a_{12} & a_{13} & \ldots & a_{1 n} \\
1 / a_{12} & 1 & a_{23} & \ldots & a_{2 n} \\
1 / a_{13} & 1 / a_{23} & 1 & \ldots & a_{3 n} \\
\vdots & \vdots & \vdots & \ddots & \vdots \\
1 / a_{1 n} & 1 / a_{2 n} & 1 / a_{3 n} & \ldots & 1
\end{array}\right)
$$

The aim is to find a positive reciprocal consistent matrix $\mathbf{X}$ in the form

$$
\mathbf{X}=\left(\begin{array}{ccccc}
1 & w_{1} / w_{2} & w_{1} / w_{3} & \ldots & w_{1} / w_{n} \\
w_{2} / w_{1} & 1 & w_{2} / w_{3} & \ldots & w_{2} / w_{n} \\
w_{3} / w_{1} & w_{3} / w_{2} & 1 & \ldots & w_{3} / w_{n} \\
\vdots & \vdots & \vdots & \ddots & \vdots \\
w_{n} / w_{1} & w_{n} / w_{2} & w_{n} / w_{3} & \ldots & 1
\end{array}\right)
$$

which minimizes the square of the Frobenius norm

$$
\|\mathbf{A}-\mathbf{X}\|_{F}^{2}=\sum_{i=1}^{n} \sum_{j=1}^{n}\left(a_{i j}-\frac{w_{i}}{w_{j}}\right)^{2},
$$

subject to

$$
\begin{aligned}
\sum_{i=1}^{n} w_{i} & =1, \\
w_{1}, w_{2}, \ldots, w_{n} & >0 .
\end{aligned}
$$

New variables $x_{1}, x_{2}, \ldots, x_{n-1}$ are introduces as follows:

$$
x_{1}=\frac{w_{1}}{w_{2}}, \quad x_{2}=\frac{w_{1}}{w_{3}}, \quad \ldots, \quad x_{i}=\frac{w_{1}}{w_{i+1}}, \quad \ldots, \quad x_{n-1}=\frac{w_{1}}{w_{n}} .
$$

The inverse formulas are computed from equations (2) and (4):

$$
w_{1}=\frac{1}{1+\sum_{j=1}^{n-1} \frac{1}{x_{j}}}, \quad w_{i}=\frac{\frac{1}{x_{i-1}}}{1+\sum_{j=1}^{n-1} \frac{1}{x_{j}}}, \quad i=2,3, \ldots, n .
$$


Based on formulas (4) matrix $\mathbf{X}$ can be written as

$$
\mathbf{X}=\left(\begin{array}{ccccc}
1 & x_{1} & x_{2} & \ldots & x_{n-1} \\
1 / x_{1} & 1 & x_{2} / x_{1} & \ldots & x_{n-1} / x_{1} \\
1 / x_{2} & x_{1} / x_{2} & 1 & \ldots & x_{n-1} / x_{2} \\
\vdots & \vdots & \vdots & \ddots & \vdots \\
1 / x_{n-1} & x_{1} / x_{n-1} & x_{2} / x_{n-1} & \ldots & 1
\end{array}\right)
$$

and the $L S M$ optimization problem (1) is reduced to

$$
\begin{gathered}
\min f\left(x_{1}, x_{2}, \ldots, x_{n-1}\right) \\
\text { s.t. } x_{1}, x_{2}, \ldots, x_{n-1}>0,
\end{gathered}
$$

where

$$
\begin{aligned}
f\left(x_{1}, x_{2}, \ldots, x_{n-1}\right) & =\|\mathbf{A}-\mathbf{X}\|_{F}^{2}=\sum_{j=2}^{n}\left[\left(a_{1 j}-x_{j-1}\right)^{2}+\left(\frac{1}{a_{1 j}}-\frac{1}{x_{j-1}}\right)^{2}\right]+ \\
& +\sum_{i=2}^{n-1} \sum_{j=i+1}^{n}\left[\left(a_{i j}-\frac{x_{j-1}}{x_{i-1}}\right)^{2}+\left(\frac{1}{a_{i j}}-\frac{x_{i-1}}{x_{j-1}}\right)^{2}\right] .
\end{aligned}
$$

The first summation in (7) comes from the first row and column of matrices $\mathbf{A}$ and $\mathbf{X}$ while the second summation corresponds to the elements of the right-down $(n-1) \times(n-1)$ submatrices. Since $f$ is a continuously differentiable function on the feasible region, the first order necessary condition of local optimality are as follows:

$$
\frac{\partial f}{\partial x_{1}}=\frac{\partial f}{\partial x_{2}}=\cdots=\frac{\partial f}{\partial x_{n-1}}=0 .
$$

The first-order partial derivatives of $f$ are rational functions of $x_{1}, x_{2}, \ldots, x_{n-1}$ and can be transformed into multivariate polynomials. One can check by using Maple that the denominator of $\frac{\partial f}{\partial x_{i}}$ has the form

$$
x_{i} \prod_{j=1}^{n-1} x_{j}^{2}, \quad i=1,2, \ldots, n-1
$$

and all the nominators of $\frac{\partial f}{\partial x_{i}}$-s $(i=1,2, \ldots, n-1)$ contain a multiplier 2 . Let $P_{1}, P_{2}, \ldots, P_{n-1}$ be defined as follows:

$$
P_{i}\left(x_{1}, x_{2}, \ldots, x_{n-1}\right)=\frac{1}{2} x_{i}\left(\prod_{j=1}^{n-1} x_{j}{ }^{2}\right) \frac{\partial f}{\partial x_{i}}, \quad i=1,2, \ldots, n-1 .
$$


The common zeros of polynomials $P_{i},(i=1,2, \ldots, n-1)$ are the solutions of the following system:

$$
\begin{array}{r}
P_{1}\left(x_{1}, x_{2}, \ldots, x_{n-1}\right)=0 ; \\
P_{2}\left(x_{1}, x_{2}, \ldots, x_{n-1}\right)=0 ; \\
P_{n-1}\left(x_{1}, x_{2}, \ldots, x_{n-1}\right)=0 .
\end{array}
$$

Since we are interested only in positive real $\left(x_{1}, x_{2}, \ldots, x_{n-1}\right)$ solutions, the systems (9) and (12) are equivalent in the sense that a positive real $(n-1)$-tuple $\left(x_{1}, x_{2}, \ldots, x_{n-1}\right)$ is a solution of (9) if and only if it is a solution of (12). This result is summarized as follows.

Proposition. All the local and global optima of the LSM problem (1) can be computed from the positive real solutions of polynomial system (12).

\section{Example 1}

Let $\mathbf{B}$ be a $4 \times 4$ pairwise comparison matrix as follows:

$$
\mathbf{B}=\left(\begin{array}{cccc}
1 & 2 & 5 & 9 \\
1 / 2 & 1 & 3 & 8 \\
1 / 5 & 1 / 3 & 1 & 4 \\
1 / 9 & 1 / 8 & 1 / 4 & 1
\end{array}\right)
$$

The aim is to approximate matrix $\mathbf{B}$ by a consistent matrix $\mathbf{X}$ in the form

$$
\mathbf{X}=\left(\begin{array}{cccc}
1 & x_{1} & x_{2} & x_{3} \\
1 / x_{1} & 1 & x_{2} / x_{1} & x_{3} / x_{1} \\
1 / x_{2} & x_{1} / x_{2} & 1 & x_{3} / x_{2} \\
1 / x_{3} & x_{1} / x_{3} & x_{2} / x_{3} & 1
\end{array}\right)
$$

The reduced $L S M$ objective function (6)-(7) is as follows:

$$
\begin{aligned}
& f\left(x_{1}, x_{2}, x_{3}\right)=\|\mathbf{B}-\mathbf{X}\|_{F}^{2}=\left(2-x_{1}\right)^{2}+\left(\frac{1}{2}-\frac{1}{x_{1}}\right)^{2}+\left(5-x_{2}\right)^{2}+\left(\frac{1}{5}-\frac{1}{x_{2}}\right)^{2}+\left(9-x_{3}\right)^{2}+ \\
& \left(\frac{1}{9}-\frac{1}{x_{3}}\right)^{2}+\left(3-\frac{x_{2}}{x_{1}}\right)^{2}+\left(\frac{1}{3}-\frac{x_{1}}{x_{2}}\right)^{2}+\left(8-\frac{x_{3}}{x_{1}}\right)^{2}+\left(\frac{1}{8}-\frac{x_{1}}{x_{3}}\right)^{2}+\left(4-\frac{x_{3}}{x_{2}}\right)^{2}+\left(\frac{1}{4}-\frac{x_{2}}{x_{3}}\right)^{2} .
\end{aligned}
$$


Multiplying the partial derivatives

$$
\begin{aligned}
& \frac{\partial f}{\partial x_{1}}=-4+2 x_{1}+\frac{2\left(\frac{1}{2}-\frac{1}{x_{1}}\right)}{x_{1}^{2}}+\frac{2\left(3-\frac{x_{2}}{x_{1}}\right) x_{2}}{x_{1}^{2}}+\frac{2\left(8-\frac{x_{3}}{x_{1}}\right) x_{3}}{x_{1}^{2}}-\frac{2\left(\frac{1}{3}-\frac{x_{1}}{x_{2}}\right)}{x_{2}}-\frac{2\left(\frac{1}{8}-\frac{x_{1}}{x_{3}}\right)}{x_{3}} ; \\
& \frac{\partial f}{\partial x_{2}}=-10+2 x_{2}-\frac{2\left(3-\frac{x_{2}}{x_{1}}\right)}{x_{1}}+\frac{2\left(\frac{1}{5}-\frac{1}{x_{2}}\right)}{x_{2}^{2}}+\frac{2\left(\frac{1}{3}-\frac{x_{1}}{x_{2}}\right) x_{1}}{x_{2}^{2}}+\frac{2\left(4-\frac{x_{3}}{x_{2}}\right) x_{3}}{x_{2}^{2}}-\frac{2\left(\frac{1}{4}-\frac{x_{2}}{x_{3}}\right)}{x_{3}} ; \\
& \frac{\partial f}{\partial x_{3}}=-18+2 x_{3}-\frac{2\left(8-\frac{x_{3}}{x_{1}}\right)}{x_{1}}-\frac{2\left(4-\frac{x_{3}}{x_{2}}\right)}{x_{2}}+\frac{2\left(\frac{1}{9}-\frac{1}{x_{3}}\right)}{x_{3}^{2}}+\frac{2\left(\frac{1}{8}-\frac{x_{1}}{x_{3}}\right) x_{1}}{x_{3}^{2}}+\frac{2\left(\frac{1}{4}-\frac{x_{2}}{x_{3}}\right) x_{2}}{x_{3}{ }^{2}}
\end{aligned}
$$

by $x_{1}^{3} x_{2}^{2} x_{3}^{2}, x_{1}^{2} x_{2}^{3} x_{3}^{2}$ and $x_{1}^{2} x_{2}^{2} x_{3}^{3}$, respectively, one gets the polynomial system of three equations and three variables:

$$
\begin{aligned}
P_{1}\left(x_{1}, x_{2}, x_{3}\right)= & -2 x_{1}{ }^{3} x_{2}{ }^{2} x_{3}{ }^{2}+x_{1}{ }^{4} x_{2}{ }^{2} x_{3}{ }^{2}+\frac{1}{2} x_{1} x_{2}{ }^{2} x_{3}{ }^{2}-x_{2}{ }^{2} x_{3}{ }^{2}+3 x_{1} x_{2}{ }^{3} x_{3}{ }^{2}-x_{2}{ }^{4} x_{3}{ }^{2}+ \\
& 8 x_{1} x_{2}{ }^{2} x_{3}{ }^{3}-x_{2}{ }^{2} x_{3}{ }^{4}-\frac{1}{3} x_{1}{ }^{3} x_{2} x_{3}{ }^{2}+x_{1}{ }^{4} x_{3}{ }^{2}-\frac{1}{8} x_{1}{ }^{3} x_{2}{ }^{2} x_{3}+x_{1}{ }^{4} x_{2}{ }^{2}=0 ; \\
P_{2}\left(x_{1}, x_{2}, x_{3}\right)= & -5 x_{2}{ }^{3} x_{1}{ }^{2} x_{3}{ }^{2}+x_{2}{ }^{4} x_{1}{ }^{2} x_{3}{ }^{2}-3 x_{1} x_{2}{ }^{3} x_{3}{ }^{2}+x_{2}{ }^{4} x_{3}{ }^{2}+\frac{1}{5} x_{2} x_{1}{ }^{2} x_{3}{ }^{2}-x_{1}{ }^{2} x_{3}{ }^{2}+ \\
& \frac{1}{3} x_{1}{ }^{3} x_{2} x_{3}{ }^{2}-x_{1}{ }^{4} x_{3}{ }^{2}+4 x_{2} x_{1}{ }^{2} x_{3}{ }^{3}-x_{1}{ }^{2} x_{3}{ }^{4}-\frac{1}{4} x_{2}{ }^{3} x_{1}{ }^{2} x_{3}+x_{2}{ }^{4} x_{1}{ }^{2}=0 \\
P_{3}\left(x_{1}, x_{2}, x_{3}\right)= & -9 x_{3}{ }^{3} x_{1}{ }^{2} x_{2}{ }^{2}+x_{3}{ }^{4} x_{1}{ }^{2} x_{2}{ }^{2}-8 x_{1} x_{2}{ }^{2} x_{3}{ }^{3}+x_{2}{ }^{2} x_{3}{ }^{4}-4 x_{2} x_{1}{ }^{2} x_{3}{ }^{3}+x_{1}{ }^{2} x_{3}{ }^{4}+ \\
& \frac{1}{9} x_{3} x_{1}{ }^{2} x_{2}{ }^{2}-x_{1}{ }^{2} x_{2}{ }^{2}+\frac{1}{8} x_{1}{ }^{3} x_{2}{ }^{2} x_{3}-x_{1}{ }^{4} x_{2}{ }^{2}+\frac{1}{4} x_{2}{ }^{3} x_{1}{ }^{2} x_{3}-x_{2}{ }^{4} x_{1}{ }^{2}=0 .
\end{aligned}
$$

Algorithms of solving polynomial systems such as the one above, as well as a few properties regarding the degrees of the polynomials are presented in the next section.

\section{Polynomial systems}

A few properties of the objective functions and the polynomial systems computed from $L S M$-problem of pairwise comparison matrices are summarized in this section.

The following definitions are used. The total/minimal degree of a multivariate polynomial can be gotten by adding the exponents of the variables in every term, and taking the maximum/minimum. For example, the polynomial $x_{1} x_{2}^{2}+x_{1}^{4} x_{2}^{3}+x_{1}^{5} x_{2}$ has total degree 7 and minimal degree 3 .

By using the symbolic computations of Maple, one can find the properties of the polynomial systems (12) as follows: 


\begin{tabular}{|c|c|c|c|c|c|c|c|c|}
\hline $\begin{array}{c}\text { Size of matrix } \\
(n \times n) \\
\end{array}$ & $n=3$ & $n=4$ & $n=5$ & $n=6$ & $n=7$ & $n=8$ & $n=9$ & $n=10$ \\
\hline $\begin{array}{l}\text { Number of terms } \\
\text { in the objective } \\
\text { function } f \\
\left(n^{2}-n\right)\end{array}$ & 6 & 12 & 20 & 30 & 42 & 56 & 72 & 90 \\
\hline $\begin{array}{l}\text { Number of terms } \\
\text { in } P_{i} \\
i=1,2, \ldots, n-1\end{array}$ & 8 & 12 & 16 & 20 & 24 & 28 & 32 & 36 \\
\hline $\begin{array}{c}\text { Degree }\left(P_{i}, x_{j}\right) \\
i=1,2, \ldots, n-1 \\
j=1,2, \ldots, n-1\end{array}$ & 4 & 4 & 4 & 4 & 4 & 4 & 4 & 4 \\
\hline $\begin{array}{c}\text { Total degree } \\
\text { of } P_{i} \\
i=1,2, \ldots, n-1 \\
\end{array}$ & 6 & 8 & 10 & 12 & 14 & 16 & 18 & 20 \\
\hline $\begin{array}{c}\text { Minimal degree } \\
\text { of } P_{i} \\
i=1,2, \ldots, n-1\end{array}$ & 2 & 4 & 6 & 8 & 10 & 12 & 14 & 16 \\
\hline
\end{tabular}

Table 1. Properties of polynomial systems, $n=3,4, \ldots, 10$.

As Table 1 shows, the polynomial systems (12) have some properties of symmetry. $\operatorname{Deg}\left(P_{i}, x_{j}\right)=4$ for any $i=1,2, \ldots, n-1, \quad j=1,2, \ldots, n-1$ indices and for any size $n$. For a fixed size $n$, both the total and the minimal degree of $P_{i}$ and the number of terms in $P_{i}$ are independent from $i$.

Polynomial systems are not easy to solve in general. The method based on Gröbner bases [7] in Maple (Groebner Package) works for the $3 \times 3$ matrices but runs out of memory as $n>3$. A method based on resultants was given for solving the $L S M$ problem for $3 \times 3$ matrices [4], and another one with Robert H. Lewis [5] based on generalized resultants for $4 \times 4$ matrices.

Homotopy continuation method is a general technique for solving nonlinear systems proposed by Drexler [15] Garcia, Zangwill [20]. Homotopy method is based on that if two non-linear systems have the same the number of roots, then the roots can be corresponded to each other and the path from a root to another one is a continuous trajectory or homotopy. The homotopy connects the nonlinear system to be solved with another system which is already solved. In the paper, the code written by Tien-Yien Li and Tangan $\mathrm{Gao}^{2}[27,19]$ is used.

\footnotetext{
${ }^{2}$ The author is very grateful to Tangan Gao for his help in using the software hom $4 p s$.
} 


\section{Numerical experiences}

This section presents the solution of Example 1 in Section 1 followed by test results based on pairwise comparison matrices of size $3 \times 3,4 \times 4, \ldots, 8 \times 8$.

The polynomial system derived from Example 1 in Section 2 has 212 solutions, 16 of which are real and there exist only one positive solution:

$$
\begin{aligned}
& x_{1}^{\star}=1.23095456 ; \\
& x_{2}^{\star}=3.88538906 ; \\
& x_{3}^{\star}=9.57889668 .
\end{aligned}
$$

Consequently, $x_{1}^{\star}, x_{2}^{\star}, x_{3}^{\star}$ is the only stationary point of reduced $L S M$ objective function of Example 1. The Hessian matrix of $f$ is positive definite in $\left(x_{1}^{\star}, x_{2}^{\star}, x_{3}^{\star}\right)$, and from formulas (5), the optimal solution of the LSM problem is

$$
\begin{aligned}
& w_{1}^{L S M}=0.460 ; \\
& w_{2}^{L S M}=0.374 ; \\
& w_{3}^{L S M}=0.118 ; \\
& w_{4}^{L S M}=0.048 .
\end{aligned}
$$

It is observed that the complexity of the $L S M$ problem arises quickly as the dimension of the matrix increases: both the number of solutions of the polynomial systems and the CPU time grows exponentially. Table 2 presents some technical information about the solutions of polynomial systems from pairwise comparison matrices of sizes $3 \times 3, \ldots, 8 \times 8$. A processor of $1 \mathrm{GHz}$ with 1 Gbyte memory is used ${ }^{3}$.

${ }^{3}$ The author thanks the National Information Infrastructure Development Program (NIIF) for the Supercomputer Service. 


\begin{tabular}{|c||c|c|c|c|c|c|}
\hline $\begin{array}{c}\text { Size of matrix } \\
(n \times n)\end{array}$ & $n=3$ & $n=4$ & $n=5$ & $n=6$ & $n=7$ & $n=8$ \\
\hline \hline CPU time & $0.05 \mathrm{sec}$ & $0.5 \mathrm{sec}$ & $20 \mathrm{sec}$ & $14 \mathrm{~min}$ & 10 hours & 3 days \\
\hline $\begin{array}{c}\text { Number of } \\
\text { common } \\
\text { roots }\end{array}$ & 24 & 224 & 1640 & $O\left(10^{4}\right)$ & $O\left(10^{5}\right)$ & $O\left(10^{6}\right)$ \\
\hline $\begin{array}{c}\text { Number of } \\
\text { real common } \\
\text { roots }\end{array}$ & $4-10$ & $8-18$ & $16-46$ & $32-76$ & $64-92$ & $128-160$ \\
\hline $\begin{array}{c}\text { Number of } \\
\text { positive real } \\
\text { common roots }\end{array}$ & $1-7$ & $1-11$ & $1-31$ & $1-15$ & $1-28$ & $1-21$ \\
\hline $\begin{array}{c}\text { Number of } \\
\text { local } \\
\text { minima }\end{array}$ & $0-1$ & $0-3$ & $0-5$ & $0-5$ & $0-7$ & $0-7$ \\
\hline $\begin{array}{c}\text { Number of } \\
\text { global } \\
\text { minima }\end{array}$ & $1-3$ & $1-4$ & $1-5$ & $1-6$ & $1-7$ & $1-8$ \\
\hline $\begin{array}{c}\text { Total } \\
\text { number of } \\
\text { minima }\end{array}$ & $1-4$ & $1-4$ & $1-10$ & $1-6$ & $1-14$ & $1-8$ \\
\hline
\end{tabular}

Table 2. Solutions of polynomial systems, $n=3,4, \ldots, 8$.

Data in Table 2 come from the experiences with some thousands of matrices, some entries might be improved by analyzing more matrices and by finding specific examples. 


\section{LSM problem for incomplete pairwise com- parison matrices}

If a decision maker has $n$ objects to compare, he/she needs to fill in $n(n-1) / 2$ elements of the upper triangular submatrix of the $n \times n$ pairwise comparison matrix. This number quickly increases by $n$, e.g., for $n=10$ the decision maker is requested to make 45 comparisons.

The Least Squares approximation of a pairwise comparison matrix has the advantage that it can be used in cases of missing elements, too. If the value in the $(i, j)$-th position of the matrix is unknown, we simply skip the corresponding term $\left(a_{i j}-\frac{w_{i}}{w_{j}}\right)^{2}$ from the objective function (1) or the term $\left(a_{i j}-\frac{x_{j-1}}{x_{i-1}}\right)^{2}$ in $(8)$.

In the case of incomplete pairwise comparison matrices, the $L S M$ problem is as follows:

$$
\begin{array}{ll}
\min & \sum_{\substack{i, j=1 \\
a_{i j} \text { is known }}}^{n}\left(a_{i j}-\frac{w_{i}}{w_{j}}\right)^{2} \\
\text { s.t. } & \sum_{i=1}^{n} w_{i}=1, \\
& w_{i}>0, \quad i=1,2, \ldots, n .
\end{array}
$$

Steps (4)-(8) may be applied also in the incomplete case with the modification that only the pairs of indices $(i, j)$ are considered in summation in (8) for which the values of $a_{i j}$ are known. Based on symbolic computations of Maple, the denominator of $\frac{\partial f}{\partial x_{i}}$ is

$$
x_{i}^{3} \cdot \prod_{\substack{j=1 \\ j \neq i \\ a_{i j} \text { is known }}}^{n-1} x_{j}{ }^{2}, \quad i=1,2, \ldots, n-1
$$

and, similarly to the complete case, all the nominators of $\frac{\partial f}{\partial x_{i}}-\mathrm{s}(i=1,2, \ldots, n-1)$ contain a multiplier 2. Consequently, the modification of step (10) is as follows:

$$
P_{i}\left(x_{1}, x_{2}, \ldots, x_{n-1}\right)=\frac{1}{2} \frac{\partial f}{\partial x_{i}} x_{i}^{3} \prod_{\substack{j=1 \\ j \neq i \\ a_{i j} \text { is known }}}^{n-1} x_{j}{ }^{2}
$$




\section{Example 2}

Let us $\mathbf{C}$ be a $4 \times 4$ incomplete pairwise comparison matrix similar to matrix $\mathbf{B}$ in Example 1 but two elements and their reciprocals are omitted:

$$
\begin{aligned}
\mathbf{C} & =\left(\begin{array}{cccc}
1 & 2 & & 9 \\
1 / 2 & 1 & 3 & \\
& 1 / 3 & 1 & 4 \\
1 / 9 & & 1 / 4 & 1
\end{array}\right) . \\
f\left(x_{1}, x_{2}, x_{3}\right)=\|\mathbf{C}-\mathbf{X}\|_{F}^{2} & =\left(2-x_{1}\right)^{2}+\left(\frac{1}{2}-\frac{1}{x_{1}}\right)^{2}+\left(9-x_{3}\right)^{2}+\left(\frac{1}{9}-\frac{1}{x_{3}}\right)^{2}+ \\
& \left(3-\frac{x_{2}}{x_{1}}\right)^{2}+\left(\frac{1}{3}-\frac{x_{1}}{x_{2}}\right)^{2}+\left(4-\frac{x_{3}}{x_{2}}\right)^{2}+\left(\frac{1}{4}-\frac{x_{2}}{x_{3}}\right)^{2} .
\end{aligned}
$$

Partial derivatives of $f$ can be written as

$$
\begin{aligned}
& \frac{\partial f}{\partial x_{1}}=-4+2 x_{1}+\frac{2\left(\frac{1}{2}-\frac{1}{x_{1}}\right)}{x_{1}^{2}}+\frac{2\left(3-\frac{x_{2}}{x_{1}}\right) x_{2}}{x_{1}^{2}}-\frac{2\left(\frac{1}{3}-\frac{x_{1}}{x_{2}}\right)}{x_{2}} ; \\
& \frac{\partial f}{\partial x_{2}}=-\frac{2\left(3-\frac{x_{2}}{x_{1}}\right)}{x_{1}}+\frac{2\left(\frac{1}{3}-\frac{x_{1}}{x_{2}}\right) x_{1}}{x_{2}{ }^{2}}+\frac{2\left(4-\frac{x_{3}}{x_{2}}\right) x_{3}}{x_{2}{ }^{2}}-\frac{2\left(\frac{1}{4}-\frac{x_{2}}{x_{3}}\right)}{x_{3}} \\
& \frac{\partial f}{\partial x_{3}}=-18+2 x_{3}-\frac{2\left(4-\frac{x_{3}}{x_{2}}\right)}{x_{2}}+\frac{2\left(\frac{1}{9}-\frac{1}{x_{3}}\right)}{x_{3}{ }^{2}}+\frac{2\left(\frac{1}{4}-\frac{x_{2}}{x_{3}}\right) x_{2}}{x_{3} 2} .
\end{aligned}
$$

After multiplication of the partial derivatives by $x_{1}^{3} x_{2}^{2}, x_{1}^{2} x_{2}^{3} x_{3}^{2}$ and $x_{2}^{2} x_{3}^{3}$ respectively (note that the multipliers are different from Example 1's), the polynomial system is

$$
\begin{aligned}
P_{1}\left(x_{1}, x_{2}, x_{3}\right)= & -2 x_{1}{ }^{3} x_{2}{ }^{2}+x_{1}{ }^{4} x_{2}{ }^{2}+\frac{1}{2} x_{1} x_{2}{ }^{2}-x_{2}{ }^{2}+3 x_{1} x_{2}{ }^{3}-x_{2}{ }^{4}-\frac{1}{3} x_{1}{ }^{3} x_{2}+x_{1}{ }^{4} \\
P_{2}\left(x_{1}, x_{2}, x_{3}\right)= & -3 x_{2}{ }^{3} x_{1} x_{3}{ }^{2}+x_{2}{ }^{4} x_{3}{ }^{2}+\frac{1}{3} x_{2} x_{1}{ }^{3} x_{3}{ }^{2}-x_{1}{ }^{4} x_{3}{ }^{2}+4 x_{2} x_{1}{ }^{2} x_{3}{ }^{3}-x_{1}{ }^{2} x_{3}{ }^{4} \\
& -\frac{1}{4} x_{2}{ }^{3} x_{1}{ }^{2} x_{3}+x_{2}{ }^{4} x_{1}{ }^{2} \\
P_{3}\left(x_{1}, x_{2}, x_{3}\right)= & -9 x_{3}{ }^{3} x_{2}{ }^{2}+x_{3}{ }^{4} x_{2}{ }^{2}-4 x_{3}{ }^{3} x_{2}+x_{3}{ }^{4}+\frac{1}{9} x_{3} x_{2}{ }^{2}-x_{2}{ }^{2}+\frac{1}{4} x_{3} x_{2}{ }^{3}-x_{2}{ }^{4} .
\end{aligned}
$$

The polynomial system above has 128 solutions, 8 of which are real and one of them is positive:

$$
\begin{aligned}
& x_{1}^{\star}=1.04817478 ; \\
& x_{2}^{\star}=2.53971708 ; \\
& x_{3}^{\star}=9.1562478 .
\end{aligned}
$$


Since the Hessian of $f$ is positive definite in $\left(x_{1}^{\star}, x_{2}^{\star}, x_{3}^{\star}\right)$, the optimal solution of the $L S M$ problem is computed from formulas (5):

$$
\begin{aligned}
& w_{1}^{L S M}=0.407 ; \\
& w_{2}^{L S M}=0.388 ; \\
& w_{3}^{L S M}=0.160 ; \\
& w_{4}^{L S M}=0.044 .
\end{aligned}
$$

The symmetrical properties regarding the number of terms and degrees of polynomial systems listed in Table 1 do not hold in the incomplete case. However, numerical experience show that homotopy method for finding the roots of the polynomial system works as well as in the case of complete matrices.

What is the optimal number of pairwise comparisons if it is less than $n(n-1) / 2$ ? It is an open question and the author's opinion is that the answer should be based on both theoretical reasoning and empirical studies from real life decision problems.

\section{Global and local optima}

In this section, two numerical examples having multiple solutions are presented. Both matrices have high inconsistency and it would be hard to find a real decision situation in which these matrices might be considered acceptable. At the same time, both have as many global optima as the dimension of the matrices, moreover, the second example has the same number of local optima, too. As it is shown, global and local optimum values may be very close to each other, which indicates the complexity of the optimization problem. 


\section{Example 3}

Let $\mathbf{D}$ be a $7 \times 7$ matrix as follows:

$$
\mathbf{D}=\left(\begin{array}{ccccccc}
1 & 8 & 1 & 1 & 1 & 1 & 1 / 8 \\
1 / 8 & 1 & 8 & 1 & 1 & 1 & 1 \\
1 & 1 / 8 & 1 & 8 & 1 & 1 & 1 \\
1 & 1 & 1 / 8 & 1 & 8 & 1 & 1 \\
1 & 1 & 1 & 1 / 8 & 1 & 8 & 1 \\
1 & 1 & 1 & 1 & 1 / 8 & 1 & 8 \\
8 & 1 & 1 & 1 & 1 & 1 / 8 & 1
\end{array}\right)
$$

There exist 7 global minimum places and the solutions have a cyclic symmetry of the weights:

$$
\begin{aligned}
\mathbf{w}^{L S M_{1}}= & (0.18757,0.08962,0.22590,0.07721,0.19463,0.09299,0.13207), \\
\mathbf{w}^{L S M_{2}}= & (0.08962,0.22590,0.07721,0.19463,0.09299,0.13207,0.18757), \\
\mathbf{w}^{L S M_{3}}= & (0.22590,0.07721,0.19463,0.09299,0.13207,0.18757,0.08962), \\
\mathbf{w}^{L S M_{4}}= & (0.07721,0.19463,0.09299,0.13207,0.18757,0.08962,0.22590), \\
\mathbf{w}^{L S M_{5}}= & (0.19463,0.09299,0.13207,0.18757,0.08962,0.22590,0.07721), \\
\mathbf{w}^{L S M_{6}}= & (0.09299,0.13207,0.18757,0.08962,0.22590,0.07721,0.19463), \\
\mathbf{w}^{L S M_{7}}= & (0.13207,0.18757,0.08962,0.22590,0.07721,0.19463,0.09299), \\
& f\left(\mathbf{w}^{L S M_{i}}\right)=342.721256 \quad \text { for } i=1,2, \ldots, 7 .
\end{aligned}
$$

The existence of multiple cyclic symmetric solutions is due to the cyclic symmetry of $\mathbf{A}$ and the 7 solutions result in 7 different ranks. In the next example, the number of minima is twice as many as the dimension of the matrix.

\section{Example 4}

Let $\mathbf{E}$ be a $5 \times 5$ matrix as follows:

$$
\mathbf{E}=\left(\begin{array}{ccccc}
1 & 6 & 1 & 1 & 1 / 6 \\
1 / 6 & 1 & 6 & 1 & 1 \\
1 & 1 / 6 & 1 & 6 & 1 \\
1 & 1 & 1 / 6 & 1 & 6 \\
6 & 1 & 1 & 1 / 6 & 1
\end{array}\right)
$$

There exist 5 global and 5 local minimum places and both groups of the 
solutions have a cyclic symmetry:

$$
\begin{aligned}
\mathbf{w}^{L S M_{1}} & =(0.28115,0.12140,0.28693,0.12389,0.18663), \\
\mathbf{w}^{L S M_{2}} & =(0.12140,0.28693,0.12389,0.18663,0.28115), \\
\mathbf{w}^{L S M_{3}} & =(0.28693,0.12389,0.18663,0.28115,0.12140), \\
\mathbf{w}^{L S M_{4}} & =(0.12389,0.18663,0.28115,0.12140,0.28693), \\
\mathbf{w}^{L S M_{5}} & =(0.18663,0.28115,0.12140,0.28693,0.12389), \\
f\left(\mathbf{w}^{L S M_{i}}\right)=126.50024 & \text { for } i=1,2, \ldots, 5, \\
\mathbf{w}^{L S M_{6}} & =(0.10697,0.21811,0.18711,0.16052,0.32730), \\
\mathbf{w}^{L S M_{7}} & =(0.21811,0.18711,0.16052,0.32730,0.10697), \\
\mathbf{w}^{L S M_{8}} & =(0.18711,0.16052,0.32730,0.10697,0.21811), \\
\mathbf{w}^{L S M_{9}} & =(0.16052,0.32730,0.10697,0.21811,0.18711), \\
\mathbf{w}^{L S M_{10}} & =(0.32730,0.10697,0.21811,0.18711,0.16052), \\
f\left(\mathbf{w}^{L S M_{i}}\right)=126.50148 & \text { for } i=6,7, \ldots, 10 .
\end{aligned}
$$

Note that the difference between the global and the local optimum values is very small (0.00124), however, the global and the local minimum places are not close to each other at all. The 5 global and 5 local minima result in 10 different ranks. Finding the reasons of having multiple global and local optima simultaneously, may be one of the interesting questions of future research.

Matrices D and E have been created based on the idea of Jensen ([24], section 8. Degeneracy and Nonuniqueness of LSM Scalings) regarding $3 \times 3$ pairwise comparison matrices. $3 \times 3$ submatrices of pairwise comparison matrices of arbitrary size, which are, themselves, pairwise comparison matrices were analyzed in details by Kéri [26].

\section{Conclusion}

A new global optimization method for solving the $L S M$ problem for pairwise comparison matrices is given in the paper. The $L S M$ optimization problem is transformed to a polynomial system which can be solved by resultant method, generalized resultant method using the computer algebra system 
Fermat implemented by Robert H. Lewis, or homotopy method, implemented by Tangan Gao and Tien-Yien Li. Present CPU and memory capacity allow us to solve the $L S M$ problem up to $8 \times 8$ matrices. One of the advantages of $L S M$ weighting method is that it can be used even if the pairwise comparison matrix is not completely filled in. The solution of $L S M$ problem is not unique in general. Numerical examples show that the number of solutions of the $L S M$ problem may be equal to or even twice as many as the dimension of the matrix.

\section{References}

[1] Barzilai, J., Cook, W.D, Golany, B. [1987]: Consistent weights for judgements matrices of the relative importance of alternatives, Operations Research Letters, 6, pp. 131-134.

[2] Blankmeyer, E., [1987]: Approaches to consistency adjustments, Journal of Optimization Theory and Applications, 54, pp. 479-488.

[3] Borda, J.C. de [1781]: Mémoire sur les électiones au scrutin, Histoire de l'Académie Royale des Sciences, Paris.

[4] Bozóki, S. [2003]: A method for solving $L S M$ problems of small size in the AHP, Central European Journal of Operations Research, 11, pp. 1733.

[5] Bozóki, S., Lewis, R. [2005]: Solving the Least Squares Method problem in the AHP for $3 \times 3$ and $4 \times 4$ matrices, Central European Journal of Operations Research, 13, pp. 255-270.

[6] Bozóki, S. [2006]: Weights from the least squares approximation of pairwise comparison matrices (in Hungarian, Súlyok meghatározása páros összehasonlítás mátrixok legkisebb négyzetes közelítése alapján), Alkalmazott Matematikai Lapok, 23, pp. 121-137.

[7] Buchberger, B. [1965]: On Finding a Vector Space Basis of the Residue Class Ring Modulo a Zero Dimensional Polynomial Ideal (in German), PhD Dissertation, University of Innsbruck, Department of Mathematics, Austria.

[8] Budescu, D.V., Zwick, R., Rapoport, A. [1986]: A comparison of the Eigenvector Method and the Geometric Mean procedure for ratio scaling, Applied Psychological Measurement, 10, pp. 69-78. 
[9] Chu, A.T.W., Kalaba, R.E., Spingarn, K. [1979]: A comparison of two methods for determining the weight belonging to fuzzy sets, Journal of Optimization Theory and Applications 4, pp. 531-538.

[10] Cook, W.D., Kress, M. [1988]: Deriving weights from pairwise comparison ratio matrices: An axiomatic approach, European Journal of Operational Research, 37, pp. 355-362.

[11] Condorcet, M. [1785]: Essai sur l'Application de l'Analyse à la Probabilité des Décisions Rendues á la Pluralité des Voix, Paris.

[12] Crawford, G., Williams, C. [1985]: A note on the analysis of subjective judgment matrices, Journal of Mathematical Psychology 29, pp. 387405.

[13] De Jong, P. [1984]: A statistical approach to Saaty's scaling methods for priorities, Journal of Mathematical Psychology 28, pp. 467-478.

[14] DeGraan, J.G. [1980]: Extensions of the multiple criteria analysis method of T.L. Saaty (Technical Report m.f.a. 80-3) Leischendam, the Netherlands: National Institute for Water Supply. Presented at EURO IV, Cambridge, England, July 22-25.

[15] Drexler, F.J. [1978]: Eine Methode zur Berechnung sämtlicher Lösungen von Polynomgleichungssystemen, Numerische Mathematik, 29, pp. 4558.

[16] Farkas, A., Lancaster, P., Rózsa, P. [2003]: Consistency adjustment for pairwise comparison matrices, Numerical Linear Algebra with Applications, 10, pp. 689-700.

[17] Fechner, G.T. [1860]: Elemente der Psychophysik, Breitkopf und Härtel, Leipzig.

[18] Fülöp, J. [2008]: A method for approximating pairwise comparison matrices by consistent matrices, Journal of Global Optimization (in print).

[19] Gao, T., Li, T.Y., Wang, X. [1999]: Finding isolated zeros of polynomial systems in $\mathbb{C}^{n}$ with stable mixed volumes, Journal of Symbolic Computation, 28, pp. 187-211.

[20] Garcia, C.B., Zangwill, W.I. [1979]: Finding all solutions to polynomial systems and other systems of equations, Mathematical Programming, 16, pp. 159-176. 
[21] Gass, S.I., Rapcsák, T. [2004]: Singular value decomposition in AHP, European Journal of Operational Research 154, pp. 573-584.

[22] Golany, B., Kress, M. [1993]: A multicriteria evaluation of methods for obtaining weights from ratio-scale matrices, European Journal of Operational Research, 69, pp. 210-220.

[23] Hashimoto, A. [1994]: A note on deriving weights from pairwise comparison ratio matrices, European Journal of Operational Research, 73, pp. 144-149.

[24] Jensen, R.E. [1983]: Comparison of Eigenvector, Least squares, Chi square and Logarithmic least square methods of scaling a reciprocal matrix, Working Paper 153 http://www.trinity.edu/rjensen/127wp/127wp.htm

[25] Jensen, R.E. [1984]: An Alternative Scaling Method for Priorities in Hierarchical Structures, Journal of Mathematical Psychology 28, pp. 317332.

[26] Kéri, G. [2005]: Criteria for pairwise comparison matrices (in Hungarian, Kritériumok páros összehasonlítás mátrixokra), Szigma, 36, pp. 139-148.

[27] Li, T.Y. [1997]: Numerical solution of multivariate polynomial systems by homotopy continuation methods, Acta Numerica, 6, pp. 399-436.

[28] Saaty, T.L. [1980]: The analytic hierarchy process, McGraw-Hill, New York.

[29] Saaty, T.L., Vargas, L.G. [1984]: Comparison of eigenvalues, logarithmic least squares and least squares methods in estimating ratios, Mathematical Modeling, 5, pp. 309-324.

[30] Thorndike, E.L. [1920]: A Constant Error in Psychological Ratings, Journal of Applied Psychology, 4, pp. 25-29.

[31] Thurstone, L.L. [1927]: The Method of Paired Comparisons for Social Values, Journal of Abnormal and Social Psychology, 21, pp. 384-400.

[32] Zahedi, F. [1986]: A simulation study of estimation methods in the Analytic Hierarchy Process, Socio-Economic Planning Sciences, 20, pp. 347354. 